\title{
Distancia social, creencias estereotípicas y emociones hacia las personas con discapacidad en universitarios mexicanos: diferencias por sexo
}

\author{
Social distance, stereotypical beliefs and emotions towards people with \\ disabilities in Mexican university students: differences by sex
}

\begin{abstract}
Resumen
Este trabajo pretende determinar las diferencias entre emociones, creencias estereotípicas e intención conductual como componentes de las actitudes hacia personas con discapacidad, en hombres y mujeres. Participaron 564 estudiantes universitarios mexicanos, 270 mujeres y 292 hombres. Se administró la Escala de Creencias Estereotípicas hacia las Personas con Discapacidad, la Escala de Emociones hacia las Personas con Discapacidad y la Escala de Distancia Social de Bogardus. Los resultados principales evidencian, en los hombres, la presencia de creencias estereotípicas negativas y una intención conductual a la exclusión. En el caso de las mujeres se evidencia la expresión de más emociones positivas y disposición a convivir con las personas que viven con discapacidad. En la discusión de resultados la construcción de género cobra relevancia y se proponen líneas de acción para avanzar en la construcción de comunidades universitarias con actitudes más positivas hacia las personas con discapacidad y hacia la inclusión.
\end{abstract}

\section{Palabras clave}

Discapacidad, creencias estereotípicas, emociones, distancia social, psicología social.

\begin{abstract}
This work aims to establish the differences between emotions, stereotypical beliefs and behavioral intention, as components of attitudes towards people with disabilities, in men and women. Participants were 564 Mexican university students, 270 women and 292 men. The Stereotypical Belief Scale for Persons with Disabilities, the Emotion Scale for Persons with Disabilities and the Bogardus Social Distance Scale were administered. The main results show, in men, the presence of negative stereotypical beliefs and a behavioral intention to exclusion. In the case of women, the expression of more positive emotions and willingness to live with people who live with disabilities is evident. In the discussion of results the construction of gender becomes relevant and action lines are proposed to advance in the construction of university communities with more positive attitudes towards people with disabilities and towards inclusion.
\end{abstract}

\section{Keywords}

Disability, stereotypical beliefs, emotions, social distance, social psychology.

\author{
Rebelín Echeverría \\ Echeverría \\ <rechever@correo.uady.mx> \\ Universidad Autónoma de Yucatán. \\ México
}

Mirta Margarita Flores Galaz <fgalaz@correo.uady.mx>

Universidad Autónoma de Yucatán. México

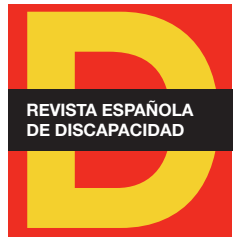

Para citar:

Echeverría, R. y Flores, M. M. (2018): "Distancia social, creencias estereotípicas y emociones hacia las personas con discapacidad en universitarios mexicanos: diferencias por sexo". Revista Española de Discapacidad, 6 (I): 221-239.

Doi: <https://doi.org/10.5569/23405104.06.01.11>

Fecha de recepción: 29-09-2017 Fecha de aceptación: 06-03-2018 


\section{Introducción}

Históricamente, la discapacidad ha sido objeto de estudio de la medicina y del área de la salud en general. Es decir, el análisis de la discapacidad tradicionalmente se ha sustentado en el modelo médico, el cual veía la discapacidad como un defecto o problema inherente a la persona, causado por enfermedad o trauma y como una desviación a ciertas normas. Bajo este modelo, las acciones se centran en la rehabilitación médica y en reformas en las políticas de salud (Soler et al, 2008).

No es hasta mediados del siglo XX cuando la discapacidad comienza a ser objeto de estudio de disciplinas sociales como la psicología y la sociología, comprendiéndola como un problema construido socialmente. Bajo este modelo social, la discapacidad deja de considerarse como un atributo de las personas y se asume como una realidad compleja creada por el ambiente social. Así, desde este modelo la discapacidad se convierte en una responsabilidad social que permita avanzar en el reconocimiento y la plena participación de las personas con discapacidad en todas las áreas de la vida. Desde esta visión, el problema requiere de un cambio social actitudinal y en la esfera política se convierte en un problema de derechos humanos (Soler et al, 2008). Los derechos humanos de la población con discapacidad deben estar dirigidos a equilibrar el acceso al ejercicio pleno de sus derechos y oportunidades en una sociedad dentro de la cual puedan desarrollar libremente y con dignidad sus planes y proyectos de vida (Victoria, 2013).

En la actualidad han sido las personas con discapacidad y sus organizaciones las que han emprendido un empoderamiento en las áreas social, política y económica (Aguilar, 2010), situación que evidencia la necesidad de incrementar los esfuerzos para promover cambios en las sociedades en general.

En este sentido, es en el año 2006 cuando la Organización de Naciones Unidas aprueba el primer tratado para los derechos de las personas con discapacidad, con la finalidad de que éstas disfruten sin discriminación de los mismos derechos y libertades en los escenarios laborales, educativos y de salud. Así, en virtud de la Convención sobre los Derechos de las Personas con Discapacidad (ONU, 2006), los gobiernos del mundo que la han ratificado han asumido la responsabilidad de garantizar que todas las personas con discapacidad disfruten de sus derechos sin discriminación alguna, reconociendo que la discriminación sobre la base de la discapacidad es una forma de opresión. Pero, sin duda alguna, de acuerdo con el Fondo de las Naciones Unidas para la Infancia (2013) la afirmación de la igualdad de derechos y la no discriminación en las leyes y las políticas tiene que acompañarse con cambios de conciencia sobre la discapacidad en las sociedades en general y entre quienes proporcionan servicios esenciales de salud, educación y protección social.

La Organización Mundial de la Salud (2011) apunta que en el mundo hay más de mil millones de personas que viven con alguna forma de discapacidad además de que, en el futuro, la discapacidad será motivo de mayor preocupación, debido a que su prevalencia está en aumento. Particularmente en México, de acuerdo con la Encuesta Nacional de la Dinámica Demográfica (ENADID) (INEGI, 2014) la prevalencia de la discapacidad es del $6 \%$, es decir, 7,1 millones de habitantes del país no pueden o tienen mucha dificultad para hacer alguna de las ocho actividades evaluadas: caminar, subir o bajar usando sus piernas; ver (aunque use lentes); mover o usar sus brazos o manos; aprender, recordar o concentrarse; escucha, $r$ (aunque use aparato auditivo); bañarse, vestirse o comer; hablar o comunicarse y problemas emocionales o mentales. 
Sin duda, el papel de la ciencia ha sido fundamental para la comprensión de la discapacidad y la generación de acciones para afrontarla con calidad. Particularmente, la psicología se ha acercado a entender la discapacidad más desde el área clínica y educativa que desde la perspectiva psicosocial. De acuerdo con Ruiz (2005) es en las dos últimas décadas cuando los psicólogos y las psicólogas se han aproximado a la comprensión de las personas con discapacidad como integrantes de un grupo estigmatizado socialmente.

De acuerdo con Ruiz y Moya (2007) las principales líneas de investigación y trabajos publicados en inglés y español en el campo de la psicología social de la discapacidad desde 1988 hacen referencia a: 1) La naturaleza de la estigmatización de las personas con discapacidad, 2) La naturaleza de la interacción entre personas con y sin discapacidad, 3) Las estrategias de afrontamiento del estigma y 4) Las estrategias que eliminan o reducen la imagen negativa de la persona con discapacidad y su estigma.

Las actitudes y expectativas negativas hacia las personas con discapacidad siguen siendo en la actualidad motivo de análisis, ya que constituyen una de las principales barreras para su inclusión e integración (Polo et al, 2011). En la actualidad y en las diferentes sociedades, independientemente de su nivel de desarrollo, las actitudes colectivas imperantes dirigidas hacia las personas con discapacidad aún tienen mucho de negativas y peyorativas (Aguado, 1995; Roeher, 1985, citado en Aguado et al, 2004; Fondo de las Naciones Unidas para la Infancia, 2013; Sevilla et al, 2014). Las actitudes suelen estar cargadas de prejuicios y mitos falsos que hacen ver a la persona con discapacidad como inferior, incompetente, incapaz e inaceptable (Ammerman, 1997; Söder, 1990). Son prejuicios y estereotipos que encajan dentro de las denominadas concepciones estigmatizantes que discapacitan más que la propia discapacidad y que justifican el mantenimiento de ciertas actitudes (Rodríguez-Bailón y Moya, 2003, Aguado et al, 2004, Soto y Vasco, 2008, citado en Delgado, 2015).

Suprimir las barreras a las que sometemos a las personas que viven con alguna discapacidad es uno de los grandes retos de nuestra sociedad. En dicho colectivo se hace más evidente la discriminación al presentarse mayor incidencia de acoso sexual, malos tratos y violencia social, así como mayores dificultades para acceder al mercado de trabajo, exclusión que se debe, más que a una limitación derivada de la discapacidad, a barreras y prejuicios existentes entre empresarios y empleados sin discapacidad (Sevilla et al, 2014). Por su parte, la violencia social no sólo se traduce en barreras arquitectónicas, sino en las derivadas de la actitud de la sociedad que con sus normas y modas estéticas les aísla, negándoles el acceso normalizado a la cultura y a la interacción personal (Fernández, 2009).

De acuerdo con Fernández (2009), con las personas con discapacidad se sigue aplicando una moral de evitación, es decir, se trata de evitarles el mal, evitarles la sensación de que se les vea como extraños. Se les hace ver, en cierto modo, que están indefensos en este mundo y que como consecuencia mejor se quedan en casa encerrados. Estas actitudes, lejos de asegurar y promover la autonomía y el bienestar integral de las personas, promueven las prácticas de dependencia, paternalismo y exclusión social.

Particularmente en México, la Primera Encuesta Nacional de Discriminación (2005) evidencia que casi el $24 \%$ de los mexicanos aceptan que las personas con alguna discapacidad son quienes sufren más por su condición y el $22 \%$ acepta que son a quienes les sería más difícil conseguir un trabajo. Sin embargo, el mexicano promedio discrimina a los discapacitados por su condición, ya que el $41 \%$ opina que los discapacitados no trabajan tan bien como el resto de las personas, el $66 \%$ está de acuerdo con que las escuelas 
donde hay muchos discapacitados disminuyen la calidad de la enseñanza y el $42 \%$ está de acuerdo con que, dadas las dificultades de empleo actuales en México, sería preferible dar trabajo a las personas sin discapacidad. Por su parte, la gran mayoría de las personas con alguna discapacidad se sientes discriminadas, casi el $100 \%$ opina que sí hay discriminación por su condición, cerca del $50 \%$ opina que no se han respetado sus derechos en México, casi el $60 \%$ se siente rechazado por la sociedad y para casi el $83 \%$ de las personas con discapacidad la discriminación hacia su condición se asocia con menos oportunidades de empleo por miedo a reducir la productividad. A casi el 40 \% le han pagado un salario menor por desempeñar un trabajo similar al de una persona sin discapacidad y al $42 \%$ le han negado el trabajo por su discapacidad. Los espacios en los que perciben mayor discriminación son el trabajo, la escuela, los hospitales públicos y la familia. Casi la mitad cree que en México tienen pocas posibilidades de mejorar sus condiciones de vida, la pobreza y la falta de trabajo son los mayores sufrimientos de las personas con discapacidad. Finalmente, para el 60 \% la discriminación ha aumentado o permanece igual en los últimos cinco años.

De acuerdo con la Encuesta Nacional sobre Discriminación en México, Enadis 2010 (2011), casi cuatro de cada diez personas de nivel socieconómico medio alto y alto consideran que los derechos de las personas con discapacidad no se respetan. Esta percepción disminuye en niveles socioeconómicos más bajos (tres de cada diez). Además de que los tres problemas que más señalan las personas con discapacidad son el desempleo, la discriminación y el no ser autosuficientes, se mencionan en menor medida los problemas relacionados con la salud, la carencia de espacios públicos adecuados y el respeto a sus derechos.

En la Encuesta sobre discriminación en la Ciudad de México (2013), de una lista de 40 grupos en situación de discriminación, las personas que viven con discapacidad ocupan el octavo lugar en experimentar mayor discriminación. El 10\% de los encuestados señalan la discapacidad como la sexta causa de discriminación y el $86 \%$ considera que existe discriminación hacia las personas con discapacidad.

En este contexto, la investigación reciente señala que las actitudes son un aspecto fundamental para el éxito de las personas con discapacidad en sus procesos de integración y en aquellos de enseñanza-aprendizaje (Bueno, 2010; Jarvis y French, 1990; Verdugo et al., 1994, citado en Araya-Cortés et al, 2014). De manera particular, la investigación de las actitudes hacia la discapacidad de los estudiantes universitarios cobra relevancia en tanto éstas pueden condicionar el grado de inclusión o exclusión que vivirá dicho colectivo. A partir de identificar las actitudes hacia las personas que viven con discapacidad en la población universitaria se puede trabajar para cambiarlas en caso de ser negativas o fortalecerlas si son positivas, de tal manera que al interior de las universidades se promueva el respecto de sus derechos y se contribuya a la formación de futuros profesionales más sensibles y promotores de la igualdad y la inclusión en todos los campos.

En México se han desarrollado diferentes estudios centrados en las actitudes hacia las personas con discapacidad. Ejemplo de ello son los trabajos de Bausela Herreras (2009) y de García y Hernández (2011). Bausela (2009) realizó una investigación con el objetivo de conocer las actitudes hacia la discapacidad que manifiesta un grupo de estudiantes de psicología de la Universidad Autónoma de San Luis Potosí. Participaron 268 estudiantes universitarios de la UASLP (México) de diferentes semestres de la licenciatura en psicología. El instrumento aplicado fue la 'Escala de Actitudes hacia las personas con discapacidad. Forma G' (Verdugo et al, 1994). Los autores sustentan que las actitudes son una de las variables más importantes para la integración de los alumnos con necesidades educativas especiales, y es que las actitudes son el primer elemento que puede facilitar o dificultar el proceso de integración e inclusión de alumnos con necesidades 
educativas especiales en la educación superior (Reina, 2003), siendo necesario contar con instrumentos válidos y fiables (Bausela, 2009).

Por su parte, García y Hernández (2011) desarrollaron un estudio cuyo objetivo fue analizar las actitudes hacia las personas con discapacidad en personas jóvenes y adultas de Chiapas. Participaron 736 personas y los instrumentos fueron la 'Escala de Valoración de Términos Asociados con la Discapacidad' (Aguado y Alcedo, 1999) y la 'Escala de Actitudes hacia las Personas con Discapacidad, Forma G.' (Verdugo et al, 1994). Los resultados indican que las variables "tener un familiar con discapacidad", "poseer una mayor escolaridad", "ser más joven", "soltero o casado", "vivir en zona urbana" y "no profesar ninguna religión" señalan actitudes más positivas hacia estas personas. Además de que no se advierten diferencias entre las calificaciones de hombres y mujeres.

Como se puede notar, el instrumento más utilizado para medir las actitudes hacia las personas con discapacidad es el de Verdugo et al, (1994) en su forma G. De manera particular, este estudio partió de conceptualizar las actitudes como integradas por un componente cognitivo, otro afectivo y finalmente, uno conductual o conativo, de acuerdo con el tradicional Modelo Tripartito que se asocia a las actitudes prejuiciosas propuesto por Allport (1954). El primero refiere al conjunto de cogniciones o creencias en torno al objeto actitudinal, es decir, un individuo puede evaluar, positiva o negativamente, a un determinado grupo porque posee un conocimiento de las características de ese grupo. El segundo corresponde al repertorio afectivo o emocional que puede activar sentimientos favorables o desfavorables hacia el objeto, es decir, una persona puede tener una actitud positiva o negativa hacia un grupo, debido a una situación vivida con algún miembro de ese grupo. El tercer componente se refiere al comportamiento de las personas ante el objeto de actitud, a la discriminación o el componente conductual del prejuicio. Los tres componentes comparten el ser evaluaciones de un mismo objeto de actitud (Morales y Moya, 1996). De ahí que se decidiera recurrir a la medición de las creencias estereotípicas (componente cognitivo), las emociones (componente afectivo) y la distancia social o intención conductual (componente conductual) como evaluadores de un mismo objeto actitudinal.

A partir de lo anteriormente mencionado, el objetivo de este trabajo es determinar las diferencias, en hombres y mujeres, entre la intención conductual, las creencias estereotípicas y las emociones como componentes de las actitudes dirigidas a las personas que viven con alguna discapacidad como uno de los grupos estigmatizados socialmente.

\section{Método}

Este estudio es de corte cuantitativo descriptivo-correlacional.

\subsection{Participantes}

La selección de la muestra se realizó mediante un muestreo no probabilístico de todas las licenciaturas que contaran con estudiantes en el último año de la carrera tanto en Mérida (zona urbana) como en el interior 
del estado (zona rural). La decisión estuvo motivada por el hecho de estar estos estudiantes próximos a graduarse y, en este sentido, tener más probabilidades de estar en contacto en su quehacer como profesionales con personas que viven con alguna discapacidad. Participaron la totalidad de los estudiantes del último año de seis licenciaturas de la ciudad de Mérida, Yucatán y cuatro licenciaturas del interior del estado, a excepción de quienes no asistieron a clases el día de la administración. La muestra quedó conformada por 564 estudiantes universitarios, de los cuales 270 fueron mujeres y 292 hombres, con un promedio de edad de 23,13 años y una desviación estándar de 2.86. En la tabla 1 se incluye la cantidad de estudiantes que participaron por programa de licenciatura, así como el porcentaje de la muestra total por sexo de cada licenciatura.

\begin{tabular}{|c|c|c|c|c|}
\hline \multicolumn{2}{|c|}{ Dependencia Universitaria } & \multirow{2}{*}{$\begin{array}{c}\begin{array}{c}\text { Número } \\
\text { Participantes }\end{array} \\
62 \\
\end{array}$} & \multirow{2}{*}{$\begin{array}{c}\begin{array}{c}\% \\
\text { Hombres }\end{array} \\
11,3(33)\end{array}$} & \multirow{2}{*}{$\begin{array}{c}\begin{array}{c}\% \\
\text { Mujeres }\end{array} \\
10,7(29)\end{array}$} \\
\hline 1. & Licenciatura en Contaduría, Centro de Estudios Superiores CTM & & & \\
\hline 2. & Licenciatura en Derecho, Centro Universitario Felipe Carrillo Puerto & 57 & $7,5(22)$ & $12,6(34)$ \\
\hline 3. & Licenciatura en Mercadotecnia, Universidad Autónoma de Yucatán & 55 & $8,2(24)$ & $11,5(31)$ \\
\hline 4. & Licenciatura en Derecho, Universidad Autónoma de Yucatán & 70 & $11,6(34)$ & $13,3(36)$ \\
\hline 5. & Ingeniería Electrónica, Instituto Tecnológico de Yucatán & 43 & $13,7(40)$ & $1,1(3)$ \\
\hline 6. & Ingeniería Mecánica, Instituto Tecnológico de Yucatán & 47 & $15,8(46)$ & $0,4(1)$ \\
\hline 7. & Licenciatura en Psicología Educativa, Instituto Cultural de Oriente & 70 & $3,8(11)$ & $21,9(59)$ \\
\hline 8. & Licenciatura en Informática, Instituto Tecnológico de Conkal & 34 & $5,1(15)$ & $7,0(19)$ \\
\hline 9. & $\begin{array}{l}\text { Licenciatura en Administración, Instituto Tecnológico Superior de } \\
\text { Valladolid }\end{array}$ & 68 & $12,3(36)$ & $11,5(31)$ \\
\hline 10. & $\begin{array}{l}\text { Ingeniería Industrial, Instituto Tecnológico Superior del Sur del } \\
\text { Estado de Yucatán }\end{array}$ & 58 & $10,6(31)$ & $10,0(27)$ \\
\hline \multicolumn{2}{|c|}{ Total } & 564 & $100(292)$ & $100(270)$ \\
\hline
\end{tabular}

Fuente: elaboración propia.

\subsection{Instrumentos}

La 'Escala de Distancia Social' se utilizó para medir la distancia social hacia las personas con discapacidad, la cual se tradujo y adaptó de la escala de distancia social diseñada originalmente por Bogardus (1947) para medir el nivel de contacto deseable con miembros de otros grupos. Dicha escala considera que la disposición a aceptar a otros en distintos niveles de proximidad constituye un indicador de distancia social hacia los grupos sociales. Según Biernat y Crandall (1993) es uno de los instrumentos más útiles y sencillos para medir las actitudes hacia los exogrupos. La distancia social se refiere al grado de contacto que considera aceptable con los miembros de un determinado grupo. Para este estudio se empleó un instrumento integrado por siete situaciones: 1. Excluiría de mi país a, 2. Excluiría de mi vecindario a, 3. Saludaría como personas conocidas a, 4. Viviría en el mismo vecindario con, 5. Trabajaría en una oficina junto a, 6 . Tendría como amigos y amigas a, 7. Cohabitaría con. Cada situación va seguida de una escala de cinco puntos, cuyos extremos indican el grado de acuerdo o desacuerdo. La consistencia interna de la prueba total fue de .62. 
En cuanto a la 'Escala de Creencias Estereotípicas hacia las Personas con Discapacidad', elaborada por Echeverría (2013), tras haberse realizado el análisis psicométrico, la escala quedó conformada por 40 adjetivos con cuatro dimensiones que explican el 55,33\% de la varianza total acumulada después de la rotación, siendo éstos:

Factor 1. Creencias Positivas sociopersonales afiliativas, ético-normativas e instrumentales: se refiere a las características positivas de la persona que le permiten el comunicarse y expresarse en sus relaciones sociales, mostrar congruencia con los valores sociopersonales apreciados desde la moral tradicional, reflejar funcionalidad en diferentes ámbitos (como el trabajo, la escuela y el hogar) y adaptarse a las reglas sociales. Este factor se integra por los siguientes reactivos: Amigables, Normales, Honestas, Amables, Trabajadoras, Atentas, Saludables, Especiales, Divertidas, Morales, Éticas, Capaces, Perseverantes, Limpias, Inteligentes, Decentes y Respetuosas (Alfa de Cronbach .92).

Factor 2. Creencias Negativas sociopersonales afiliativas, instrumentales e indeseabilidad social: se refiere a las características negativas de la persona que le dificultan el comunicarse y expresarse en sus relaciones sociales, que le impiden reflejar funcionalidad en diferentes ámbitos (como el trabajo, la escuela y el hogar) y que le dificultan el adaptarse a las reglas sociales. Además, hace referencia a las evaluaciones negativas que la sociedad hace al comportamiento de la persona. Este factor se integra por los siguientes reactivos: Corrientes, Un estorbo, Insensibles, Ignorantes, Motivo de burla, Antipáticas, Analfabetas, Raras, Vergüenza Familiar, Aburridas, Flojas, Indecentes y Anormales (Alfa de Cronbach .87).

Factor 3. Creencias Negativas ético-normativas: se refiere a las características negativas de la persona que le dificultan el mostrar congruencia con los valores sociopersonales apreciados desde la moral tradicional y el adaptarse a las reglas sociales. Este factor se integra por los siguientes reactivos: Incumplidas, Irrespetuosas, Antisociales, Inmorales, Sucias, Mal ejemplo y Egoístas (Alfa de Cronbach .81).

Factor 4. Aceptación Social: se refiere a las evaluaciones positivas que la sociedad hace al comportamiento de la persona. Este factor se integra por los siguientes reactivos: Aceptadas, Respetadas y Aprobadas (Alfa de Cronbach .65).

La consistencia interna de la prueba total fue de .89 .

Sobre la 'Escala de Emociones hacia las personas con discapacidad', originalmente elaborada por Rueda y Navas (1996), para este estudio se adaptó la redacción acorde a la evaluación de las personas con discapacidad. Esta escala está conformada por un listado de 16 emociones (7 positivas y 9 negativas). En este estudio se realizó la validación psicométrica por medio del análisis factorial exploratorio. La escala quedó conformada por dos factores que explican el 54,30 \% de la varianza total acumulada después de la rotación, siendo éstos: F1 Emociones Positivas (satisfacción, alegría, amor, orgullo, placer, confianza interés y serenidad) (alfa de Cronbach .84) y F2 Emociones negativas coléricas (repulsión, enojo, miedo y rabia) (alfa de Cronbach .71). La consistencia interna de la escala completa tuvo un alpha de Cronbach de .75.

Finalmente, además de las instrucciones y reactivos de cada una de las escalas se incluyeron preguntas sociodemográficas como edad, sexo y religión, así como reactivos de respuesta múltiple en cuanto a la pertenencia o identidad con algún otro grupo estigmatizado socialmente. De manera adicional, se incluye- 
ron reactivos de jerarquización u ordenamiento centrados en la medición de la presencia y percepción de discriminación en Yucatán hacia diferentes grupos estigmatizados socialmente.

\subsection{Procedimiento}

Para el desarrollo de este estudio se sometió el proyecto a aprobación del comité de investigación. Particularmente, para la aplicación de la escala se solicitó la autorización del personal directivo de las universidades seleccionadas, la cual fue oficializada mediante un documento de parte de la universidad. A los estudiantes se les explicó el objetivo del estudio y se les solicitó su cooperación voluntaria para la aplicación de la prueba, se les garantizó la confidencialidad y el anonimato, y se les señaló que podrían abandonar la investigación en el momento que así lo decidieran. Es importante destacar que ningún estudiante decidió no participar o abandonó la investigación antes de finalizarla.

La administración de las escalas tuvo una duración de 30 minutos y se realizó en las aulas de cada una de las universidades participantes, tanto en la ciudad de Mérida como en los diferentes municipios del interior del Estado. Todos los participantes contestaron todas las escalas, las cuales se ordenaron mediante azar aleatorio por tipo de instrumento.

\section{Resultados}

\subsection{Distancia social hacia personas con discapacidad por sexo}

Para la escala de distancia social se encontraron diferencias estadísticamente significativas entre hombres y mujeres en seis de los siete reactivos que conforman la escala. Se encontraron diferencias estadísticamente significativas en el reactivo 1. Cohabitaría con $\left(_{(559)}=-2.01 ; p=.005\right)$ en donde las mujeres $(M=4.47)$, presentaron una media más alta; en el reactivo 2. Tendría como amigos y amigas a $\left(t_{(560)}=-2.55 ; p=.000\right)$ también las mujeres presentaron la media más alta $(M=4.80)$; en el reactivo 3 . Trabajaría en una oficina junto a $\left(t_{(560)}=-2.45 ; p=.000\right)$ de igual manera, las mujeres obtuvieron la media más alta $(M=4.77)$; en el reactivo 4. Viviría en el mismo vecindario a $\left(\mathrm{t}_{(560)}=-1.41 ; \mathrm{p}=.012\right)$, las mujeres obtuvieron la media más alta $(M=4.84)$; en el reactivo 6 Excluiría de mi vecindario a $\left(t_{(560)}=3.13 ; p=.000\right)$, por el contrario los hombres son los que obtuvieron la media más alta $(M=1.33)$; en el reactivo 7 Excluiría de mi país a $\left(t_{(560)}=2.39 ; p=\right.$ .000 ) de igual manera, en este reactivo los hombres son los que obtuvieron la media más alta $(M=1.21)$. En este sentido, se puede decir que las mujeres manifiestan estar más dispuestas a tener amigos y trabajar con personas con discapacidad, además de estar menos dispuestas a excluirlas del vecindario y del país, a diferencia de los hombres. 


Tabla 2. Análisis de diferencias por sexo para los factores de la escala de distancia social hacia las personas con
discapacidad
\begin{tabular}{|l|c|c|c|c|c|}
\hline Reactivos & $\begin{array}{c}\text { M } \\
\text { Hombres }\end{array}$ & $\begin{array}{c}\text { M } \\
\text { Mujeres }\end{array}$ & $\boldsymbol{t}$ & gl & Prob. \\
\hline 1. Cohabitaría con & 4.30 & 4.47 & -2.01 & 559 & $.005^{\star \star}$ \\
\hline 2. Tendría como amigos y amigas a & 4.65 & 4.80 & -2.55 & 560 & $.000^{\star * *}$ \\
\hline 3. Trabajaría en una oficina junto a & 4.63 & 4.77 & -2.45 & 560 & $.000^{\star * *}$ \\
\hline 4. Viviría en el mismo vecindario con & 4.77 & 4.84 & -1.41 & 560 & $.012^{\star *}$ \\
\hline 5. Saludaría como personas conocidas a & 4.81 & 4.85 & -.86 & 559 & .104 \\
\hline 6. Excluiría de mi vecindario a & 1.33 & 1.13 & 3.13 & 558 & $.000^{\star \star *}$ \\
\hline 7. Excluiría de mi país a & 1.21 & 1.08 & 2.39 & 560 & $.000^{\star \star *}$ \\
\hline
\end{tabular}

${ }^{*} \mathrm{p} \leq .05^{* *} \mathrm{p} \leq .01{ }^{* * *} \mathrm{p} \leq .001$

Fuente: elaboración propia.

\subsection{Creencias estereotípicas hacia personas con discapacidad por sexo}

Se realizó un análisis de diferencias por sexo por medio de la Prueba t de Student para cada uno de los factores de la escala de creencias estereotípicas individuales hacia personas con discapacidad, encontrándose diferencias significativas en dos factores: en el Factor 2 relativo a Creencias Negativas Sociopersonales afiliativas, instrumentales e indeseabilidad social $\left(t_{(552.48)}=3.85 ; p=.000\right)$ en donde los hombres $(M=2.03)$, presentaron una media más alta. Es decir, los hombres en comparación con las mujeres presentaron más creencias negativas hacia las personas con discapacidad, los ven como corrientes, un estorbo, insensibles, ignorantes, motivo de burla, antipáticas, analfabetas, raras, vergüenza familiar, aburridas, flojas, indecentes $y$ anormales. En el Factor 3 sobre Creencias Negativas ético-normativas $\left(t_{(558.57)}=4.13 ; p=.000\right)$ de igual manera los hombres obtienen la media más alta $(\mathrm{M}=2.02)$. Es decir, los hombres ven a las personas con discapacidad como incumplidas, irrespetuosas, antisociales, inmorales, sucias, mal ejemplo y egoístas.

Tabla 3. Análisis de diferencias por sexo para los factores de la escala de creencias individuales hacia las personas con discapacidad

\begin{tabular}{|l|c|c|c|c|c|}
\hline Factores & $\begin{array}{c}\text { M } \\
\text { Hombres }\end{array}$ & $\begin{array}{c}\text { M } \\
\text { Mujeres }\end{array}$ & $\boldsymbol{t}$ & gl & Prob. \\
\hline $\begin{array}{l}\text { Factor 1 Creencias Positivas Sociopersonales } \\
\text { afiliativas, ético-normativas e instrumentales }\end{array}$ & 3.37 & 3.48 & -1.63 & 560 & .103 \\
\hline $\begin{array}{l}\text { Factor 2 Creencias Negativas Sociopersonales } \\
\text { afiliativas, instrumentales e indeseabilidad } \\
\text { social }\end{array}$ & 2.03 & 1.79 & 3.85 & 552.48 & $.000^{\star \star *}$ \\
\hline Factor 3 Creencias Negativas ético-normativas & 2.02 & 1.75 & 4.13 & 558.57 & $.000^{\star * *}$ \\
\hline Factor 4 Aceptación Social & 2.88 & 2.86 & .207 & 534.35 & .836 \\
\hline
\end{tabular}

${ }^{*} \mathrm{p} \leq .05^{* *} \mathrm{p} \leq .01{ }^{* \star *} \mathrm{p} \leq .00$

Fuente: elaboración propia. 


\subsection{Emociones hacia personas con discapacidad por sexo}

Para esta escala los resultados obtenidos mostraron diferencias estadísticamente significativas en el factor: I Emociones Positivas $\left(t_{(560)}=-5.47 ; p=.000\right)$ en donde las mujeres $(M=3.22)$, presentaron una media más alta. Es decir, las mujeres presentaron más emociones positivas (amor, satisfacción, confianza, alegría, orgullo, interés, serenidad, placer, preocupación) hacia las personas con discapacidad.

\begin{tabular}{|c|c|c|c|c|c|}
\hline Factores & $\begin{array}{c}\text { M } \\
\text { Hombres }\end{array}$ & $\begin{array}{c}\text { M } \\
\text { Mujeres }\end{array}$ & $t$ & gl & Prob. \\
\hline Factor I Emociones positivas & 2.82 & 3.22 & -5.47 & 560 & $.000^{\star \star \star}$ \\
\hline Factor II Emociones negativas coléricas & 1.75 & 1.74 & .227 & 560 & .820 \\
\hline
\end{tabular}

${ }^{*} \mathrm{p} \leq .05^{* *} \mathrm{p} \leq .01{ }^{* * *} \mathrm{p} \leq .001$

Fuente: elaboración propia.

Para conocer la relación entre la distancia social, las creencias estereotípicas y las emociones se obtuvo la correlación Producto Momento de Pearson entre cada una de ellas. Es importante señalar que el análisis se realizó para hombres y mujeres.

3.4. Relación entre la distancia social y las creencias estereotípicas hacia las personas con discapacidad por sexo

En la tabla 5 se puede apreciar, en cuanto a la relación entre la distancia social y las creencias estereotípicas, que las creencias positivas que muestran una disposición a cohabitar $(r=.141 ; p=.05)$ y a tener como amigo $(r=.121 ; p=.05)$, son las únicas correlaciones significativas para el caso de los hombres. Es decir, los hombres que presentan creencias positivas tienden a presentar más disposición a cohabitar o a tener como amigo a una persona que vive con discapacidad. Para el caso de las mujeres no se presenta ninguna correlación entre la distancia social y las creencias positivas.

Por otra parte, se encontró que las creencias negativas correlacionan con la distancia social en todos los casos, para ambos sexos. Para los hombres se encontró que las creencias negativas correlacionaron de manera negativa con la intención conductual a cohabitar $(r=-.204 ; p=.01)$, a tener como amigo ( $r=$ -.276; $p=.01)$, a trabajar en la misma oficina $(r=-.264 ; p=.01)$, a vivir en el mismo vecindario $(r=-.233$; $p=.01$ ) y a saludar como conocida a una persona que vive con discapacidad $(r=-.246 ; p=.01)$. Es decir, los hombres que presentan creencias negativas tienden a presentar menor intención conductual a tratar como conocidos, a vivir en el mismo vecindario, a trabajar en la misma oficina, a tener un amigo o amiga y estar dispuesto a cohabitar con alguna persona que vive con discapacidad. Adicionalmente, se encontró una relación estadísticamente significativa positiva entre las creencias negativas y la disposición a excluir del vecindario $(r=.249 ; p=.01)$ y la disposición a excluir del país $(r=.164 ; p=.01$ es decir, los hombres que 
presentan creencias negativas tienden a presentar mayor intención conductual a excluir de su vecindario o del país a personas que viven con discapacidad.

Para las mujeres se encontró que las creencias negativas correlacionan de manera negativa con la intención conductual a cohabitar ( $r=-.229 ; p=.01)$, a tener como amigo $(r=-.266 ; p=.01)$, a trabajar en la misma oficina $(r=-.231 ; p=.01)$, a vivir en el mismo vecindario $(r=-.316 ; p=.01)$ y a saludar como conocida a una persona que vive con discapacidad $(r=-.311 ; p=.01)$. Es decir, las mujeres, al igual que los hombres que presentan creencias negativas, tienden a presentar menor intención conductual a tratar como conocidos, a vivir en el mismo vecindario, a trabajar en la misma oficina, a tener un amigo o amiga y estar dispuesto a cohabitar con alguna persona que vive con discapacidad. Adicionalmente, se encontró una relación estadísticamente significativa positiva entre las creencias negativas y la disposición a excluir del vecindario ( $r=$ .312; $p=.01$ ) y la disposición a excluir del país ( $r=.312 ; p=.01)$. Es decir, las mujeres que presentan creencias negativas tienden a presentar mayor intención conductual a excluir de su vecindario o del país a personas que viven con discapacidad.

\begin{tabular}{|c|c|c|c|c|}
\hline & \multicolumn{2}{|c|}{ Creencias positivas } & \multicolumn{2}{|c|}{ Creencias negativas } \\
\hline & H & $\mathbf{M}$ & $\mathbf{H}$ & $\mathbf{M}$ \\
\hline Cohabitaría & $.141^{\star}$ & .028 & $-.204^{\star \star}$ & $-.229^{\star \star}$ \\
\hline Tendría como amigo & $.121^{\star}$ & .022 & $-.276^{\star \star}$ & $-.266^{\star \star}$ \\
\hline Trabajaría & .047 & .007 & $-.264^{\star *}$ & $-.231^{\star \star}$ \\
\hline Viviría & .074 & -.080 & $-.233^{\star *}$ & $-.316^{\star \star}$ \\
\hline Saludaría como conocido & -.033 & -.076 & $-.246^{\star \star}$ & $-.311^{\star \star}$ \\
\hline Excluiría del vecindario & .078 & .067 & $.249^{\star *}$ & $.312^{\star *}$ \\
\hline Excluiría del país & .052 & .052 & $.164^{\star *}$ & $.312^{\star *}$ \\
\hline
\end{tabular}

${ }^{*} p \leq .05^{* *} p \leq .01^{* * *} p \leq .001$

Fuente: elaboración propia.

\subsection{Relación entre las emociones y la distancia social hacia las personas con discapacidad por sexo}

En la tabla 6 se puede observar que, tanto en hombres como en mujeres, las emociones positivas correlacionaron de manera positiva con la disposición a cohabitar, a tener como amigo, a trabajar en la misma oficina, a vivir en el mismo vecindario y a saludar como conocida a una persona que vive con discapacidad. Es decir, las personas que presentan emociones positivas tienden a presentar mayor intención conductual a tratar como conocidos, a vivir en el mismo vecindario, a trabajar en la misma oficina, a tener un amigo o amiga y estar dispuesto a cohabitar con alguna persona que vive con discapacidad.

Por otra parte, se encontró que las emociones negativas correlacionaron de manera negativa con la disposición a cohabitar, a tener como amigo y a trabajar en la misma oficina, tanto para hombres como para 
mujeres. Es decir, las personas que presentan emociones negativas tienden a presentar menor intención conductual a trabajar en la misma oficina, a tener un amigo o amiga y estar dispuesto a cohabitar con alguna persona que vive con discapacidad. Para el caso de las mujeres, las emociones negativas correlacionaron de manera negativa con la disposición a vivir en el mismo vecindario ( $r=-.124 ; p=.05)$.

Finalmente, para los hombres se encontraron correlaciones positivas entre las emociones negativas y la intención conductual a excluirlos del vecindario $(r=.223 ; p=.000)$ y con excluirlos del país $(r=.126 ; p=.05)$. Es decir, las personas que presentan emociones negativas tienden a presentar mayor intención conductual a excluir del vecindario y del país a las personas que viven con discapacidad.

\begin{tabular}{|c|c|c|c|c|}
\hline & \multicolumn{2}{|c|}{ Emociones positivas } & \multicolumn{2}{|c|}{$\begin{array}{c}\text { Emociones negativas } \\
\text { Coléricas }\end{array}$} \\
\hline & $\mathbf{H}$ & $\mathbf{M}$ & $\mathbf{H}$ & M \\
\hline Cohabitaría & $.167^{\star \star}$ & $.249^{\star \star \star}$ & $-.116^{\star}$ & $-.163^{\star \star}$ \\
\hline Tendría como amigo & $.203^{\star \star \star}$ & $.257^{\star \star \star}$ & $-.216^{\star \star \star}$ & $-.167^{\star \star}$ \\
\hline Trabajaría & $.191^{\star \star \star}$ & $.260^{\star \star \star}$ & $-.172^{\star *}$ & $-.159^{\star \star}$ \\
\hline Viviría & $.231^{\star \star \star}$ & $.182^{\star \star}$ & -.097 & $-.124^{\star}$ \\
\hline Saludaría como conocido & $.200^{\star \star \star}$ & $.168^{\star \star}$ & -.035 & -.101 \\
\hline Excluiría del vecindario & -.050 & -.071 & $.223^{\star \star \star}$ & .097 \\
\hline Excluiría del país & -.047 & .047 & $.126^{\star}$ & .093 \\
\hline
\end{tabular}

${ }^{*} \mathrm{p} \leq .05^{* \star} \mathrm{p} \leq .01^{\star * \star} \mathrm{p} \leq .001$

Fuente: elaboración propia.

3.6. Relación entre las creencias estereotípicas y las emociones hacia las personas con discapacidad por sexo

En la tabla 7 se puede notar que, tanto para hombres como para mujeres, existen correlaciones positivas entre el Factor 1 Creencia Positivas Sociopersonales afiliativas, ético-normativas e instrumentales y las emociones positivas. Es decir, quienes presentan dichas creencias tienden a presentar emociones positivas. Para el Factor 2 Creencias Negativas Sociopersonales afiliativas, instrumentales e indeseabilidad social se encontró una correlación negativa con las emociones positivas, para el caso de las mujeres $(r=-.150 ; p$ $=.05$ ) y se encontraron correlaciones positivas para ambos sexos con las emociones negativas. Es decir, quienes presentan creencias negativas sociopersonales tienden a sentir emociones negativas hacia las personas con discapacidad. Por su parte el Factor 3 Creencias Negativas ético-normativas correlaciona negativamente con las emociones positivas y correlaciona positivamente con las emociones negativas coléricas, tanto para hombres como mujeres. Es decir, quienes presentan creencias negativas ético-normativas tienden a sentir emociones negativas hacia las personas con discapacidad. Finalmente, el Factor 4 Aceptación Social correlaciona positivamente con las emociones positivas solo para el caso de los hombres ( $r=$ $.167 ; p=.01)$. 


\begin{tabular}{|c|c|c|c|c|}
\hline & \multicolumn{2}{|c|}{ Emociones positivas } & \multicolumn{2}{|c|}{$\begin{array}{c}\text { Emociones negativas } \\
\text { coléricas }\end{array}$} \\
\hline & $\mathbf{H}$ & M & $\mathbf{H}$ & M \\
\hline $\begin{array}{l}\text { Factor } 1 \text { Creencias Positivas Sociopersonales afiliativas, } \\
\text { ético-normativas e instrumentales }\end{array}$ & $.376^{\star \star \star}$ & $.285^{\star \star \star}$ & .071 & .030 \\
\hline $\begin{array}{l}\text { Factor } 2 \text { Creencias Negativas Sociopersonales afiliativas, } \\
\text { instrumentales e indeseabilidad social }\end{array}$ & -.143 & $-.150^{\star}$ & $.223^{\star \star \star}$ & 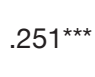 \\
\hline Factor 3 Creencias Negativas ético-normativas & $-.160^{* *}$ & $-.128^{\star *}$ & $.226^{\star \star \star}$ & $.154^{\star *}$ \\
\hline Factor 4 Aceptación Social & $.167^{\star \star}$ & .100 & .050 & -.039 \\
\hline
\end{tabular}

${ }^{*} \mathrm{p} \leq .05^{* *} \mathrm{p} \leq .01{ }^{* \star *} \mathrm{p} \leq .001$

Fuente: elaboración propia.

\section{Discusión}

Los resultados mostraron que, en la escala de creencias estereotípicas hacia la discapacidad, los hombres presentaron más creencias negativas hacia dicho grupo. En la escala de emociones, son las mujeres quienes presentaron más emociones positivas hacia las personas con discapacidad. Y finalmente, en la escala de distancia social, son las mujeres quienes manifiestan estar más dispuestas a la convivencia cercana con personas con discapacidad y menos dispuestas a la exclusión.

Algunas conclusiones apuntan que, con respecto a las creencias estereotípicas hacia las personas con discapacidad, en esta investigación se encuentran resultados similares a los reportados por Polo et al. (2011), Suriá (2011), Lissi et al. (2009), Moreno et al (2006), Gómez e Infante (2004) y Upton y Harper (2002), en tanto las actitudes suelen ser positivas o favorables por parte de estudiantes universitarios hacia personas con discapacidad. Sin embargo, a diferencia de este estudio y de otras investigaciones como las realizadas por Rizzo y Vispoel (1991), Duchane y French (1998), Iglesias y Polanco (2009), y Moreno et al. (2006), quienes no encuentran diferencias por sexo, en esta investigación sí se observan diferencias significativas en cuanto a las actitudes ante las personas con discapacidad en función del sexo.

Existen trabajos como los desarrollados por Findler et al. (2007), Upton y Harper (2002) y Aloia et al. (1980) que sustentan que las mujeres tienen una mayor aceptación y actitud positiva que los hombres hacia las personas con discapacidad. Una interpretación de estos resultados se sustenta en que se ha asociado a las mujeres, desde los procesos de construcción de género, con características como el ser más protectoras de las personas desvalidas, maternales y sensibles (Hernández et al. , 1998), además de que se las ha retratado como cálidas y orientadas a la gente (Williams y Best, 1990). 
De manera adicional, los resultados evidencian la existencia de una relación positiva entre el tener creencias positivas hacia las personas con discapacidad, sentir emociones positivas y la disposición a cohabitar, además de una relación negativa entre las creencias positivas y la intención de cohabitar, de tener amigos, de trabajar en la misma oficina, de vivir en el mismo vecindario y de saludar a alguna persona con discapacidad, y una relación positiva entre creencias negativas, emociones negativas y disposición a excluir del vecindario y país.

De acuerdo con Espinosa et al., (2007) las emociones son conceptualizadas como patrones adaptativos básicos. Las emociones básicas influyen directamente en lo que es percibido por los sentidos, afectando todo el procesamiento de información subsiguiente y las acciones derivadas del mismo (Izard, 1991). En este sentido, las respuestas emocionales frente a personas de distintos grupos sociales estarán mediadas por la empatía o preocupación por los demás (Espinosa et al., 2007).

De acuerdo con los resultados obtenidos en esta investigación, las personas que presentan emociones positivas tienden a presentar mayor intención conductual a tratar como conocidos, a vivir en el mismo vecindario, a trabajar en la misma oficina, a tener un amigo o amiga y estar dispuesto a cohabitar con alguna persona que vive con discapacidad. También tienden a presentar menor intención conductual a excluir de su vecindario a personas que viven con discapacidad. Por otra parte, las personas que presentan emociones negativas tienden a presentar mayor intención conductual a excluir del vecindario y del país a las personas que viven con discapacidad y menor intención conductual para cohabitar, tener como amigo y trabajar en la misma oficina que alguna persona que vive con discapacidad. Destaca que la presencia de emociones negativas coléricas se relaciona con la intención conductual a la exclusión. Es interesante destacar que el tener emociones positivas disminuye la disposición a excluir, sin importar el sexo.

Para el caso de los hombres, las creencias positivas se relacionan con la disposición a cohabitar y a tener amigos con discapacidad. Los hombres y mujeres que presentan creencias negativas tienden a presentar menor intención conductual a convivir y mayor intención conductual a excluir a las personas con discapacidad.

Los hombres que presentan emociones negativas coléricas tienden a presentar mayor intención conductual a excluir a personas que viven con discapacidad. Entre las consecuencias psicológicas de la exclusión cabe mencionar, según un conjunto de expertos consultados por Tezanos (1999), la pérdida de la autoestima, la ruptura de los vínculos entre el individuo y la sociedad, el deterioro físico y psíquico, así como un aumento de la agresividad y las relaciones violentas. Asimismo, desde un punto de vista psicosocial, los grupos excluidos tienen muchas probabilidades de desarrollar una identidad social negativa y de ser estigmatizados (Molero et al, 2001). De acuerdo con Arteaga y Lara (2004), es primordial analizar los condicionantes estructurales que favorecen la existencia de actitudes negativas, analizar la forma en que los distintos grupos le dan sentido a las desigualdades sociales, así como evaluar el papel de las instituciones para la reproducción de las actitudes, sean éstas positivas o negativas.

Entendiendo que las actitudes sean constructos psicosociales sobre los que una formación transversal puede incidir positivamente (Moneo y Anaut, 2017), sin duda la formación de los futuros profesionales para la superación de toda creencia estereotípica, emociones negativas e intenciones conductuales de exclusión dirigida hacia las personas con discapacidad cobra relevancia central en tanto son ellos quienes 
materializarán o harán posible la inclusión o la exclusión social. Desde un modelo social, el problema de la discapacidad no está en el individuo sino en la sociedad que lo rodea, por lo que se requiere de procesos de rehabilitación de una sociedad, que ha de ser concebida y diseñada para hacer frente a las necesidades de todas las personas, gestionando las diferencias e integrando la diversidad (Victoria, 2013). Así, podemos estar de acuerdo con Moneo y Anaut (2017) cuando afirman que el reto de la inclusión está presente tanto dentro como fuera de las universidades y, por tanto, la formación en materia de discapacidad a lo largo de la vida académica es la base para facilitar la igualdad de oportunidades, la no discriminación y la inclusión social. Dicha formación contribuiría a que las personas con discapacidad puedan ejercer su derecho a la educación y satisfacer las necesidades de su aprendizaje, considerando el contexto social y favoreciendo la integración con las personas que las rodean, de tal manera que se elimine la marginación y discriminación (García, 2013).

En futuros trabajos sería importante estudiar las actitudes positivas y negativas hacia las personas con discapacidad en muestras más amplias y con la inclusión de otras variables que han resultado relevantes en otros estudios realizados en diferentes partes del mundo como el género, la edad, el tipo de formación profesional, así como el contacto con personas que viven con alguna discapacidad y las actitudes hacia personas con discapacidades específicas. Además de que, de acuerdo con Garzón et al. (2016), con la finalidad de lograr una educación de calidad para todos en contextos inclusivos, es importante que también el personal docente tenga actitudes positivas hacia las personas con discapacidad y la inclusión, reconociendo la diferencia y la diversidad como valores de la educación.

A partir de los resultados obtenidos en esta investigación se recomienda desarrollar diversas líneas de trabajo como el diseñar e implementar programas socioeducativos que contribuyan a la reducción de actitudes negativas (creencias estereotípicas negativas, emociones negativas e intenciones de exclusión) hacia las personas con discapacidad y hacia los diversos grupos sociales que han sido y continúan siendo estigmatizados y excluidos en la sociedad actual. Lo anterior se sustenta en que existen trabajos que ponen en evidencia que el recibir información a lo largo de la formación universitaria podría ser uno de los aspectos determinantes en cuanto a las actitudes (Reina, 2003), reconociendo que las actitudes negativas son el primer elemento que puede facilitar o dificultar el proceso de integración e inclusión de alumnos con necesidades educativas especiales en educación superior, siendo necesario contar con instrumentos válidos y fiables (Bausela, 2009). De acuerdo con Ocampo (2010), habrá que reflexionar sobre las barreras simbólicas e interpretativas-culturales presentes en cada institución de educación superior, ya que éstas podrían favorecer o dificultar la puesta en marcha de estrategias que contribuyan activamente al ejercicio del derecho de estudiantes en situación de discapacidad. De acuerdo con Cruz y Casillas (2017) existen instituciones de educación superior en México donde por política institucional se ha creado un programa específico en atención a los alumnos con discapacidad, con objetivos precisos dirigidos a la inclusión de este grupo. Ejemplo de ello son la Universidad Nacional Autónoma de México, la Universidad Veracruzana, la Universidad Autónoma de Tlaxcala, la Universidad Autónoma de San Luis Potosí y la Universidad Autónoma de Nuevo León. Sin duda, se requiere de la suficiente sensibilidad en la gestión de las instituciones de educación superior para la adopción de medidas formativas dirigidas a toda la comunidad universitaria (Rodríguez-Martín y Álvarez-Arregui, 2015). 


\section{Referencias bibliográficas}

Aguado, A. L. (1995): Historia de las deficiencias. Madrid: Escuela Libre Editorial. Colección Tesis y Proxis.

Aguado, A. L. y Alcedo, M. A. (1999): "La Escala de Valoración de Términos Asociados con Discapacidad en una muestra de EGB". Análisis y Modificación de Conducta, 25 (103): 783-806.

Aguado, A. L. et al. (2004): "Programas de cambio de actitudes ante la discapacidad". Psicothema, 16 (4): $667-$ 673.

Aguilar, M. L. (2010): “Discapacidad: entre el estigma y la comunidad”. Integra educativa, IV (2): 205-216.

Allport, G. W. (1954): The nature of Prejudice. Oxford: Addison-Wesley.

Aloia, G. F. et al. (1980): "Physical education teachers' initial perceptions of handicapped children". Mental Retardation, 18 (1): 85-87.

Ammerman, R. T. (1997): "Nuevas tendencias en investigación sobre discapacidad". En II Jornadas Científicas de Investigación sobre Personas con Discapacidad, Conferencia llevada a cabo en la Universidad de Salamanca.

Araya-Cortés, A. et al. (2014): "Actitud de universitarios hacia las personas con discapacidad”. Educación y Educadores, 17 (2): 289-305.

Arteaga, N. y Lara, V. L. (2004): “Violencia y distancia social: una revisión”. Papeles de Población, 10 (40): 169-191.

Bausela, H. E. (2009): "Actitudes hacia la discapacidad: estudio de algunas propiedades psicométricas en una muestra de universitarios mexicanos”. Revista Iberoamericana de Educación, 49 (6): 1-9.

Biernat, M. y Crandall, C. S. (1993): "Racial Attitudes”, en Robinson, J. P. et al. (eds.): Measures of Political Attitudes. San Diego: Academic press.

Bogardus, E. S. (1947): "Measurement of Personal-Group Relations”. Sociometry, 10 (4): 306-311.

Bueno, A. (2010): "Una mirada ilusionada al futuro de los servicios de apoyo a la discapacidad en la Universidad. Buenas prácticas de apoyo a la discapacidad en la universidad", en Bueno, A.: Buenas prácticas durante los estudios universitarios en la recepción de información (tesis doctoral). Editorial CEE Limencop, Alicante.

Cruz, R. y Casillas, M. (2017): "Las instituciones de educación superior y los estudiantes con discapacidad en México". Revista de la Educación Superior, 46 (181): 37-53.

Delgado, A. (2015): "Estrategias psicosociales para el cambio de actitud hacia personas discriminadas por su discapacidad o trastorno mental". Revista Española de Discapacidad, 3 (2): 27-39.

Duchane, K. A. y French, R. (1998): "Attitudes and grading practices of secondary physical educators in regular education settings”. Adapted Physical Activity Quarterly, 15: 370-380.

Echeverría, R. (2013): Prejuicios de universitarios hacia las personas indígenas mayas (tesis de doctorado). Centro de Estudios, Clínica e Investigación Psicológica, Campeche (México).

Encuesta Nacional sobre Discriminación en México, Enadis 2010 (2011): Resultados Generales. México: Consejo Nacional Para Prevenir la Discriminación.

Encuesta sobre discriminación en la Ciudad de México (2013): Encuesta en viviendas Principales resultados. Consejo para Prevenir y Eliminar la Discriminación en la Ciudad de México, México. 
Espinosa, A. et al. (2007): "Estereotipos, prejuicios y exclusión social en un país multiétnico: el caso peruano". Revista de Psicología, XXV (2): 295-338.

Fernández, P. (2009): Compendio sobre violencia de género y factores de discriminación en la mujer con discapacidad. Valencia: Editorial Tirant lo Blanch.

Findler, L. et al. (2007): "The multidimensional attitudes scale toward persons with disabilities (MAS)". Rehabilitation Counseling Bulletin, 50: 166-177.

García, M. T. (2013): Estudiantes con discapacidad en la Universidad de Guadalajara, México (tesis no publicada). Universidad de Oviedo.

García, L. G. A. y Hernández, O. S. (2011): "Actitudes hacia la discapacidad de jóvenes y adultos de Chiapas”. Universitas Psychologica, 10 (3): 817-827.

Garzón, P. et al. (2016): "Inclusión educativa. Actitudes y estrategias del profesorado". Revista Española de Discapacidad, 4 (2): 25-45.

Gómez, V. e Infante, M. (2004): “Actitudes de los estudiantes de educación hacia la integración de personas con discapacidad y hacia la educación multicultural”. C\&E: Cultura y Educación, 4 (16): 371-383.

Hernández, R. et al. (1998): Metodología de la investigación. México: Mc Graw Hill.

Iglesias, D. E. y Polanco, V. (2009): Determinantes de la actitud hacia la discapacidad de personas sin discapacidad en pequeñas empresas. Caracas: Universidad Católica Andrés Bello.

Instituto Nacional de Estadística y Geografía (2014): Encuesta Nacional de la Dinámica Demográfica. México: INEGI.

Izard, C. E. (1991): The psychology of emotions. Nueva York: Plenum.

Jarvis, C. y French, R. (1990): "Attitudes of physical educators toward the integration of handicapped students". Perceptual and motor skills, 70: 899-902.

Lissi, M. et al. (2009): “Inclusión y discapacidad en contextos universitarios: la experiencia del PIANE-UC”. Revista Calidad en la Educación (CSE), (30): 306-324.

Molero, F. et al. (2001): "Inmigración, prejuicio y exclusión social: reflexiones en torno a algunos datos de la realidad española". International Journal of Psychology and Psychological Therapy, 1 (1): 11-32.

Morales, J. F. y Moya, M. C. (1996): Tratado de Psicología Social. Volumen I: Procesos básicos. Madrid: Síntesis.

Moneo, B. A. y Anaut, S. (2017): "Inclusión del alumnado con discapacidad en los estudios superiores. Ideas y actitudes del colectivo estudiantil”. Revista Española de Discapacidad, 5 (2): 129-148.

Moreno, F. J. et al. (2006): "Actitudes ante la discapacidad en el alumnado universitario matriculado en materias afines". Revista Iberoamericana de Educación, 40 (5): 1-12.

Ocampo, A. (2010): Hacia la Reivindicación de la Escuela Inclusiva. Sevilla: Asociación Andaluza por la Innovación Educativa.

Organización de Naciones Unidas, ONU (2006): Convención sobre los derechos de las personas con discapacidad (en línea). <http://www.un.org/esa/socdev/ enable/documents/tccconvs.pdf >, acceso 28 de mayo de 2018.

Organización Mundial de la Salud, OMS (2011): Informe Mundial sobre la Discapacidad. Malta: OMS-Banco Mundial. 
Polo, M. T. et al. (2011): "Estudio de las actitudes de estudiantes de Ciencias Sociales y Psicología: relevancia de la información y contacto con personas discapacitadas". Universitas Psychologica, 10 (1): 113-123.

Primera Encuesta Nacional de Discriminación en México (2005): Primera Encuesta Nacional de Discriminación en México. México: Secretaría de Desarrollo Social-Consejo Nacional para Prevenir la Discriminación.

Reina, R. (2003): "Propuesta de intervención para la mejora de actitudes hacia personas con discapacidad a través de actividades deportivas y recreativas". Revista digital, 9 (59).

Rizzo, T. L. y Vispoel, W. P. (1991): "Physical educators' attributes and attitudes toward teaching students with handicaps". Adapted Physical Activity Quarterly, 8: 4-11.

Rodríguez-Bailón, R y Moya Morales, M. (2003): "La diferencia percibida en valores como proceso vinculado, a las relaciones intergrupales de payos y gitanos". Psicothema, 15 (2): 176-182.

Rodríguez-Martín, A. y Álvarez-Arregui, E. (2015): "Universidad y discapacidad, Actitudes del profesorado y de estudiantes". Perfiles Educativos, XXXVII (147): 86-102.

Rueda, J. F. y Navas, M. (1996): "Hacia una evaluación de las nuevas formas de prejuicio racial: Las actitudes sutiles del racismo". Revista de Psicología Social, 11 (2): 131-149.

Ruiz, J. (2005): "La Discapacidad como estigma: un análisis psicosocial del afrontamiento del empleo de las personas con discapacidad física". REDSI: Revista especializada en formación y empleo de los colectivos en riesgo de exclusión, 6: 1-27.

Ruiz, J. y Moya, M. (2007): "El estudio de la discapacidad física desde la Psicología Social”. Revista de Psicología Social, 22 (2): 177-198.

Sevilla, D. E. et al. (2014): "Formación laboral como proceso de inclusión de personas con discapacidad intelectual”, en Cárdenas Aguilar, T. J. y Barraza Macías, A. (comps.): Los sujetos de la educación especial: alumnos, maestros y autoridades. Investigación en México. Durango: Universidad Pedagógica de Durango y Red Durango de Investigadores Educativos.

Soder, M. (1990): "Prejudice or Ambivalence? Attitudes toward Persons with Disabilities". Handicap and Society, 5 (3): 227-241.

Soler, A. et al. (2008): "Discapacidad y dependencia: Una perspectiva de género". En XI Jornadas de Economía Crítica, eco cri 2008. Ponencia llevada a cabo en las Jornadas de la Universidad de Granada, PUC Minas Gerais y Universitàt de València, Santa Fe (Argentina).

Suriá, R. (2011): "Comparative analysis of student's attitudes toward their classmates with disabilities". Electronic Journal of Research in Educational Psychology, 9: 197-216.

Tezanos, J. F. (1999): Tendencias en desigualdad y exclusión social: Tercer Foro sobre Tendencias Sociales. Madrid: Sistema.

UNICEF (2013): Estado Mundial de la Infancia 2013 Niñas y niños con discapacidad. Nueva York: Fondo de las Naciones Unidas para la Infancia (UNICEF).

Upton, T. y Harper, D. (2002): "Multidimensional disability attitudes and equitable evaluation of educational accommodations by college students without disabilities". Journal of Postsecondary Education and Disability, 15: $115-130$.

Verdugo, M. A. et al. (1994): Actitudes hacia las personas con minusvalía. Madrid, Ministerio de Asuntos Sociales, Instituto Nacional de Servicios Sociales. 
Distancia social, creencias estereotípicas y emociones hacia las personas con

Victoria, J.A. (2013): Hacia un modelo de atención a la discapacidad basado en los derechos humanos. Boletín Mexicano de Derecho Comparado, XLVI (138): 1093-1109.

Williams, J. E. y Best, D. L. (1990): Measuring Sex Stereotypes: a Multination Study. Newbury Park: Sage. 